\title{
Modal Decomposition of Self-Focusing Effects in Optical Fibres
}

\author{
G. Hesketh, F. Poletti, P. Horak \\ Optoelectronics Research Centre, University of Southampton, Southampton SO17 1BJ, United Kingdom
}

When an intense beam with a transverse intensity gradient propagates in a medium with positive Kerr nonlinearity $\mathrm{n}_{2}$, the induced refractive index profile actuates a lens, focusing the beam itself. For input powers above a critical threshold, $\mathrm{P}>\mathrm{P}_{\text {crit }}$, self-focusing can subjugate beam divergence, resulting in catastrophic collapse and material failure [1]. For $\mathrm{P}<\mathrm{P}_{\text {crit }}$ diffraction deflects energy from the focal point, and in a fibre, divergence is reflected at the core/cladding boundary instigating a periodic transverse beam profile.

We model self-focusing in a modal decomposition framework, wherein spatial oscillations correspond to power transfer between different modes through four-wave mixing (FWM) [2], see Figs. 1(a)-(b). The resulting multi-mode generalised nonlinear Schrödinger equation (MM-GNLSE) [3] can be simulated numerically orders of magnitude faster than propagation based slowly-varying envelope equation methods (SEE) [1] because of the relatively small number of modes involved, typically six to 10 . In addition, symmetries are easily incorporated, facilitating, for example, simulation of high order mode pumping. Moreover, modal decomposition, in principle, comfortably delivers stationary solutions where nonlinear effects are balanced to provide phase locked modal propagation, as indicated by the dashed lines in Figs. 1(a)-(b).

The MM-GNLSE also allows for extremely efficient simulation of ultrashort pulse propagation in the presence of self-focusing effects. This model incorporates all the propagation effects known from standard single-mode fibres, such as high-order material and waveguide dispersion, Raman response and self-steepening effects [3], and thus allows for the interpretation of self-focusing in terms of conventional fibre optics. As an example, we show in Fig. 1(c) ultrashort pulse propagation with a peak power below $\mathrm{P}_{\text {crit }}$ along a short silica rod in air, a waveguide that shares many properties with the microstructured fibres used for supercontinuum generation (SCG). As in the $\mathrm{cw}$ case, inter-modal power transfer initially drives periodic spatial oscillations occuring predominantly near the temporal peak. Since the spatially compressed pulse has a higher nonlinearity than the fundamental mode, we observe stronger self-phase modulation than in simulations that neglect higher order modes, thereby accelerating temporal nonlinear dynamics. Indicatively, simulation of only the fundamental mode exhibits broad SCG driven by a conventional modulation instability process, while the MM-GNLSE predicts that under the same launch conditions a singularity is reached both in time and space, cf. bottom plot of Fig. 1(c) and inset, respectively. More detailed investigations affirm, in concurrence with [4], that the temporal singularity is conceived through the interplay of self-steepening and FWM. The most notable finding is that such catastrophic temporal and spatial singularities can occur even for powers significantly below $\mathrm{P}_{\text {crit }}$.

We will discuss in detail the dynamics of ultrahigh power pulse propagation in the modal picture and consequences for nonlinear fibre optics, in particular for SCG. The dependence on fibre design, dispersion, pulse lengths and pulse shapes will be investigated as well as high order mode pumping.
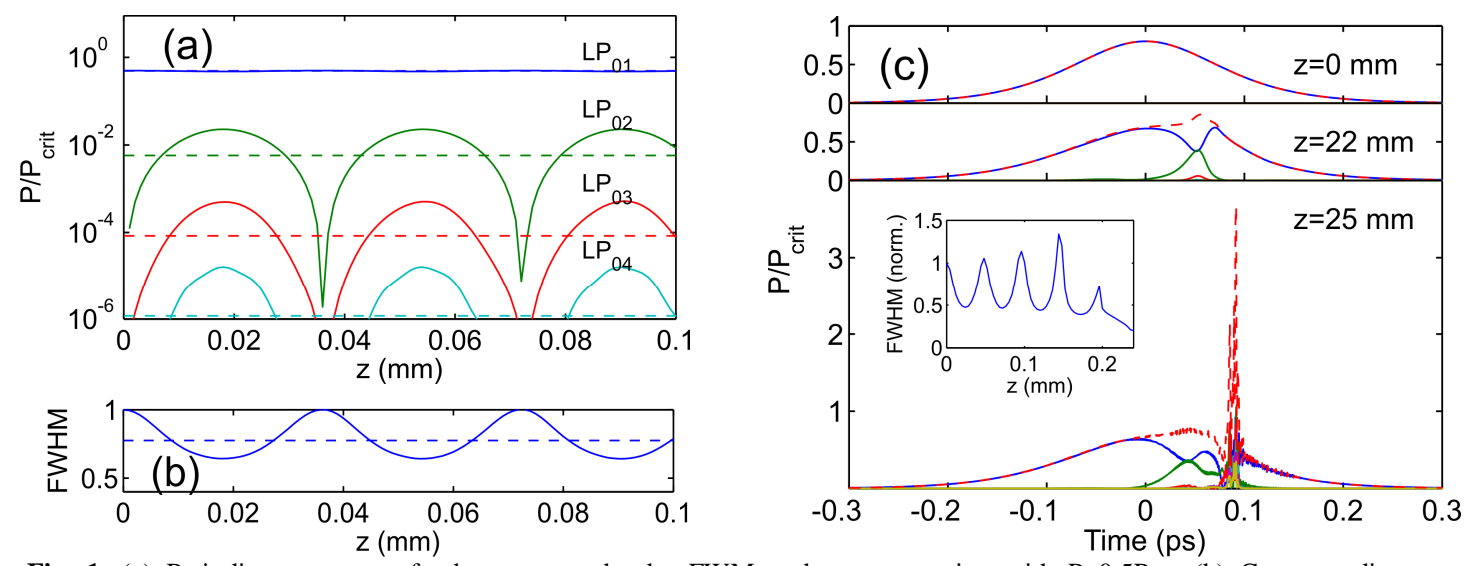

Fig. 1. (a) Periodic power transfer between modes by FWM under cw pumping with $P=0.5 \mathrm{P}_{\text {crit }}$. (b) Corresponding normalised beam diameter oscillations. (c) Modal and temporal evolution of a propagating 100 fs pulse with $\mathrm{P}=0.8 \mathrm{P}_{\text {crit }}$ launched peak power exhibiting catastrophic collapse; dashed line is the sum over all modes. The fibre is a $5 \mu \mathrm{m}$ diameter silica rod in air, supporting six $\mathrm{LP}_{0 \mathrm{n}}$ modes with a zero-dispersion wavelength of the fundamental mode at $1034 \mathrm{~nm}$. Pump wavelength is $1060 \mathrm{~nm}, \mathrm{P}_{\text {crit }}=4.6 \mathrm{MW}$.

\section{References}

[1] A. Gaeta, "Catastrophic collapse of ultrashort pulses", Phys. Rev. Lett. 84, 3582 (2000).

[2] G. Tempea, T. Brabec, "Theory of self-focusing in a hollow waveguide", Opt. Lett. 23, 762 (1998).

[3] F. Poletti, P. Horak, "Description of ultrashort pulse propagation in multimode optical fibers", JOSAB 25, 1645 (2008).

[4] N.A. Zharova et al., "Self-focusing of wave packets and envelope shock formation in nonlinear dispersive media" JETP 103, 15 (2006). 